\title{
Article \\ Serum Concentrations of Antigen-Specific IgG4 in Patients with Japanese Cedar Pollinosis
}

\author{
Shiori Kitaya ${ }^{1,2}$, Nobuo Ohta ${ }^{2, *}$, Atsushi Yuta ${ }^{3,4}$, Yukiko Ogawa ${ }^{3}$, Yusuke Suzuki ${ }^{5}$, Seiya Ichihara ${ }^{2}$, \\ Ryoukichi Ikeda ${ }^{1}$, Tadao Enomoto ${ }^{6}$, Hideaki Kouzaki ${ }^{4}{ }^{D}$, Takeshi Shimizu ${ }^{4}$ (D) Junya Ono ${ }^{7}$, Kenji Izuhara ${ }^{8}(\mathbb{D}$ \\ and Yoshitaka Okamoto ${ }^{9}$
}

check for updates

Citation: Kitaya, S.; Ohta, N.; Yuta, A.; Ogawa, Y.; Suzuki, Y.; Ichihara, S.; Ikeda, R.; Enomoto, T.; Kouzaki, H.; Shimizu, T.; et al. Serum Concentrations of Antigen-Specific IgG4 in Patients with Japanese Cedar Pollinosis. Allergies 2021, 1, 140-149. https://doi.org/10.3390/ allergies1030013

Academic Editors:

Hideyuki Kawauchi,

Emmanuel Prokopakis and

Pierre Rougé

Received: 27 March 2021

Accepted: 7 July 2021

Published: 14 July 2021

Publisher's Note: MDPI stays neutral with regard to jurisdictional claims in published maps and institutional affiliations.

Copyright: (c) 2021 by the authors. Licensee MDPI, Basel, Switzerland. This article is an open access article distributed under the terms and conditions of the Creative Commons Attribution (CC BY) license (https:// creativecommons.org/licenses/by/ $4.0 /)$.
1 Department of Otolaryngology, Head and Neck Surgery, Tohoku University Hospital 1-1, Seiryomachi, Aoba-ku, Sendai 980-8574, Japan; kitayan728@gmail.com (S.K.); ryoukich@hotmail.com (R.I.)

2 Division of Otolaryngology, Tohoku Medical and Pharmaceutical University Hospital 1-12-1, Fukumuro, Miyagino-ku, Sendai 983-8512, Japan; 21723097@is.tohoku-mpu.ac.jp

3 Yuta Clinic, 2-3 Syuseicho, Tsu 514-0837, Japan; yuta-a@pearl.ocn.ne.jp (A.Y.); yukiko-33@tg.commufa.jp (Y.O.)

4 Department of Otolaryngology, Head and Neck Surgery, Shiga University of Medical Science, Setatsukinowacho, Otsu 520-2192, Japan; hkohzaki@belle.shiga-med.ac.jp (H.K.); shimizu@belle.shiga-med.ac.jp (T.S.)

5 Department of Otolaryngology, Head and Neck Surgery, Yamagata University Faculty of Medicine, 2-2-2 Iidanishi, Yamagata 990-9585, Japan; y-suzuki@med.id.yamagata-u.ac.jp

6 Department of Otolaryngology, Head and Neck Surgery, Tottori University Faculty of Medicine, 86 Nishimachi, Yonago 683-8503, Japan; t-enomoto@tempo.ocn.ne.jp

7 Shino-Test Corporation, 2-29-14 Onodai Minami-ku, Sagamihara 252-0331, Japan; junya.ono@shino-test.co.jp

8 Division of Medical Biochemistry, Department of Biomolecular Sciences, Saga Medical School, 5-1-1 Nabeshima, Saga 849-8501, Japan; kizuhara@cc.saga-u.ac.jp

9 Department of Otolaryngology, Head and Neck Surgery, Graduate School of Medicine Chiba University, 1-8-1 Inohana, Chuo-ku, Chiba 260-8670, Japan; yokamoto@faculty.chiba-u.jp

* Correspondence: noohta@hosp.tohoku-mpu.ac.jp; Tel.: +81-022-259-1221

Abstract: Purpose: To elucidate the usefulness of Japanese cedar pollen (JCP)-specific antigen-specific immunoglobulin (IgG) 4 as a biomarker for predicting the efficacy of sublingual immunotherapy for cedar pollen-induced allergic rhinitis. Methods: We divided a total of 105 cases with Japanese cedar pollinosis into three groups: "SLIT Successful," SLIT Unsatisfactory," and "SCIT" groups. The SLIT group patients were treated with JCP Droplet (Torii Pharmaceutical Co. Ltd., Tokyo, Japan) for one year from 2015 and were divided into two groups, the SLIT Successful group or the SLIT Unsatisfactory group. The SLIT Successful group $(n=16)$ were subjects treated by SLIT only, who were able to experience control of their naso-ocular symptoms without the need for antiallergic rescue agents during the peak season of atmospheric pollen. The SLIT Unsatisfactory group $(n=76)$ comprised subjects treated with SLIT only, who did not respond successfully, and were administered with rescue agents to control their naso-ocular symptoms. The SCIT group had been treated with standardized JCP extract (Torii Pharmaceutical Co., Ltd., Tokyo, Japan) for three years from 2012, and were also able to experience control of their symptoms during the peak pollen season without the need for antiallergic rescue agents. We determined the serum level of JCP-specific immunoglobulin $\mathrm{E}$ (IgE), IgG, and IgG4 used in the 3gAllergy-specific IgE assay (3gAllergy). The serum levels of periostin and SCCA2 were measured using established ELISA procedures (clones SS18A and SS17B; Shino-Test, Japan) following the manufacturer's instructions. We then made ROC curves for each group and assessed which index was best able to predict the efficacy of sublingual immunotherapy. Results: Serum JCP-specific IgE was significantly lower in the SCIT group than in the SLIT Successful group and the SLIT Unsatisfactory group $(p<0.05)$. Serum JCP-specific IgG was significantly higher in the SCIT group and the SLIT Successful group than in the SLIT Unsatisfactory group $(p<0.05)$. Serum JCP-specific IgG4 was also significantly higher in the SCIT group and the SLIT Successful group than in the SLIT Unsatisfactory group $(p<0.05)$. There was no significant difference among serum levels of periostin in the SCIT group, the SLIT Successful group, or the SLIT Unsatisfactory group. There was also no significant difference in SCCA2 among the three groups. In terms of ROC curves, a serum JCP-specific IgG4 value greater than $989.5 \mathrm{UA} / \mathrm{mL}$ showed the best sensitivity (93.3\%) 
and specificity $(94.7 \%)(p<0.05)$ among other parameters. Conclusions: The serum JCP-specific IgG4 level is significantly correlated with the clinical efficacy of SLIT. Serum JCP-specific IgG4 cutoff levels greater than $989.5 \mathrm{UA} / \mathrm{mL}$ were correlated with an effective clinical response to SLIT, with a sensitivity of $93.3 \%$ and a specificity of $94.7 \%$.

Keywords: sublingual immunotherapy; Japanese cedar pollinosis; IgG4; subcutaneous immunotherapy; periostin

\section{Introduction}

The prevalence of Japanese cedar pollinosis (JCP) is rising rapidly in Japan. Pharmacotherapy is currently the commonest treatment for patients with this condition; however, its clinical benefits are occasionally limited because some patients demonstrate persistent tissue inflammation despite repeated high doses of glucocorticoid (GC) treatment [1]. Allergen immunotherapy (AIT) is the only treatment modality with the potential to both relieve naso-ocular symptoms and improve patients' quality of life. Sublingual immunotherapy (SLIT), which is regarded as a safe and effective treatment for patients with JCP, has recently been developed [2,3]. However, useful biomarkers for measuring the mechanism and efficacy of AIT have yet to be established [4]. Antigen-specific immunoglobulin (IgG) 4 is a blocking antibody that may serve as a useful marker. House dust mite-specific IgG4 is useful for predicting the effects of subcutaneous immunotherapy (SCIT) [4,5]; however, it has only rarely been applied to investigations of cedar pollen allergy, still less of SLIT. The usefulness of JCP-specific IgG4 and periostin in the management of JCP is still controversial, so to clarify this question, we performed a prospective study to investigate the serum concentrations of JCP-specific IgG4, JCP-specific IgE, squamous cell carcinoma antigen A2 (SCCA2), and periostin in patients with JCP pollinosis who had received AIT.

\section{Materials and Methods}

\subsection{Patients}

All patients were treated by experienced ENT doctors at Shiga University of Medical Science, Yuta Clinic, and at Tohoku Medical and Pharmaceutical University between October 2014 and August 2015. Informed consent was obtained under protocols approved by the Institutional Review Board (2018-2-139). A retrospective survey of medical records identified 105 patients (50 men and 55 women, average age 41.7). We divided the total of 105 cases with Japanese cedar pollinosis into three groups: SLIT Successful, SLIT Unsatisfactory, or SCIT groups. The details of each group's clinical background are shown in Table 1. SLIT group patients were treated with JCP Droplet (Torii Pharmaceutical Co., Ltd., Tokyo, Japan) for one year from 2015, and were divided into two groups: the SLIT Successful group or the SLIT Unsatisfactory group. The SLIT Successful group $(n=16)$ comprised subjects treated by SLIT only, who were able to experience control of their nano-ocular symptoms without any need for antiallergic rescue agents during the peak season of scattered pollen. The SLIT Unsatisfactory group $(n=76)$ were subjects treated with SLIT only, who did not respond successfully and were therefore administered with rescue agents to control their naso-ocular symptoms. The SCIT group $(n=13)$ had been treated with standardized JCP extract (Torii Pharmaceutical Co. Ltd., Tokyo, Japan) for three years from 2012, who were also able to experience control of symptoms without antiallergic rescue agents during the peak pollen season. All the serum samples were collected during the peak season of Japanese cedar pollen release in 2015. 
Table 1. Clinical characteristics.

\begin{tabular}{ccccc}
\hline & $\begin{array}{c}\text { SLIT Successful } \\
(\boldsymbol{n}=\mathbf{1 6})\end{array}$ & $\begin{array}{c}\text { SLIT Unsatisfactory } \\
(\boldsymbol{n}=\mathbf{7 6})\end{array}$ & $\begin{array}{c}\text { SCIT } \\
(\boldsymbol{n}=\mathbf{1 3})\end{array}$ & Significance \\
\hline Sex (male:female), $n$ & $8: 8$ & $36: 40$ & $6: 7$ & $\mathrm{~ns}$ \\
Age at onset, years, median (range) & $30.2(12-56)$ & $28.9(11-62)$ & $32.1(12-55)$ & $\mathrm{ns}$ \\
Age at enrolment, years, median (range) & $42.1(17-64)$ & $40.6(18-71)$ & $41.9(21-59)$ & $\mathrm{ns}$ \\
Comorbidity of CRSwNP, $n(\%)$ & $1(6.1)$ & $2(8.9)$ & $0(0)$ & $\mathrm{ns}$ \\
Comorbidity of asthma, $n(\%)$ & $2(12.5)$ & $10(13.2)$ & $2(15.4)$ & $\mathrm{ns}$ \\
Comorbidity of allergic conjunctivitis, $n(\%)$ & $16(100)$ & $74(97.4)$ & $13(100)$ & $\mathrm{ns}$ \\
Area (eastern:western), $n$ & $4: 12$ & $19: 57$ & $\mathrm{~ns}$ & $\mathrm{~ns}$ \\
\hline
\end{tabular}

Chronic rhinosinusitis with nasal polyp, CRSwNP ICS; eastern area of Japan, eastern; western area of Japan, western; ns: not significant.

\subsection{Measurement Methods}

Immunoglobulin Measurements

We measured the serum level of JCP-specific immunoglobulin E (IgE), IgG, and IgG4 used in the 3gAllergy-specific IgE assay (3gAllergy). The serum levels of periostin and SCCA2 were measured using established ELISA procedures (clones SS18A and SS17B; Shino-Test, Sagamihara Japan), following the manufacturer's instructions [6,7].

\subsection{Assessment of Symptoms and Use of Medication}

The effectiveness of SLIT/SCIT was evaluated based on the subjects' clinical allergic symptoms such as lacrimation, runny nose, and sneezing. We then placed these patients in the SLIT Successful group if patients showed their symptoms to be under control without the use of antiallergic rescue agents.

\subsection{Statistical Analysis}

We used one-way analysis of variance (ANOVA) and the Mann-Whitney U test to identify any statistical differences between groups. The analysis was performed using SPSS version 20 statistical software (IBM, Chicago, IL, USA). Differences in corrected $p$-value of $<0.05$ were regarded as significant. Whether SLIT was effective or not was assessed by examining the area under the receiver operating characteristic (ROC) curve. Models with an ROC area of at least 0.80 were considered to have predictive value.

\section{Results}

\subsection{Serum JCP-Specific Levels of IgE, IgG, and IgG4}

Serum JCP-specific IgE was significantly lower in the SCIT group than in the SLIT Successful group and the SLIT Unsatisfactory group $(p<0.05$; Figure $1 \mathrm{~A})$. Serum JCPspecific IgG was significantly higher in the SCIT group and the SLIT Successful group than in the SLIT Unsatisfactory group ( $p<0.05$; Figure 1B). Serum JCP-specific IgG4 was also significantly higher in the SCIT group and the SLIT Successful group than in the SLIT Unsatisfactory group $(p<0.05$; Figure 1C). 


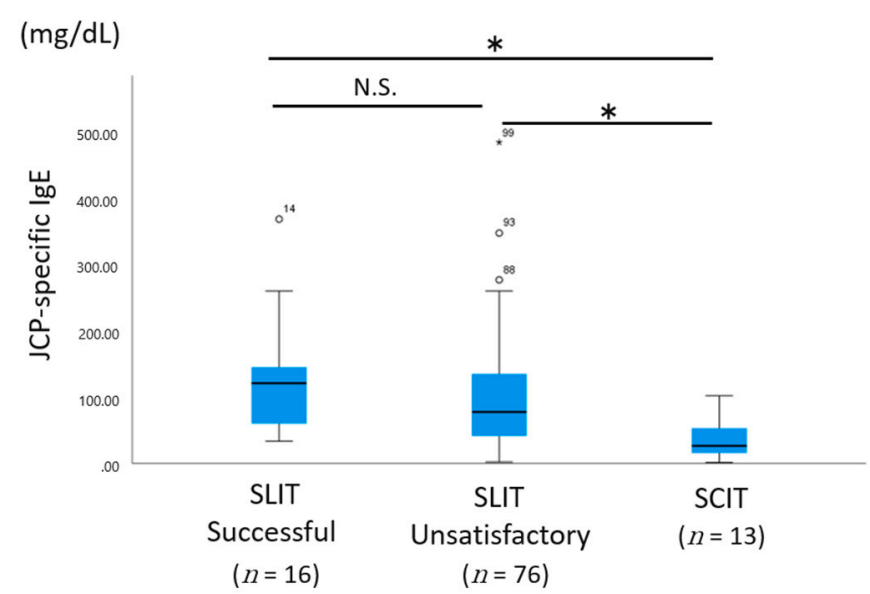

(A)

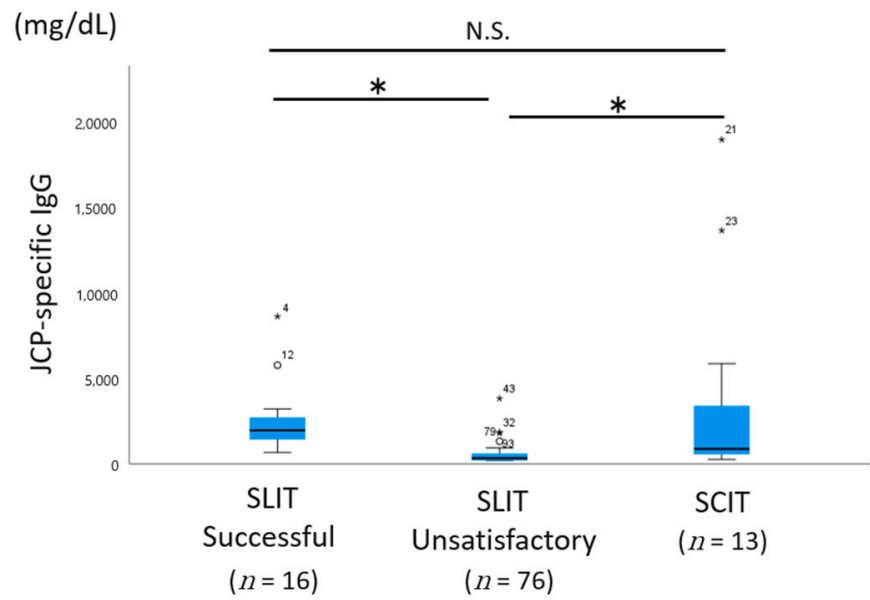

(B)

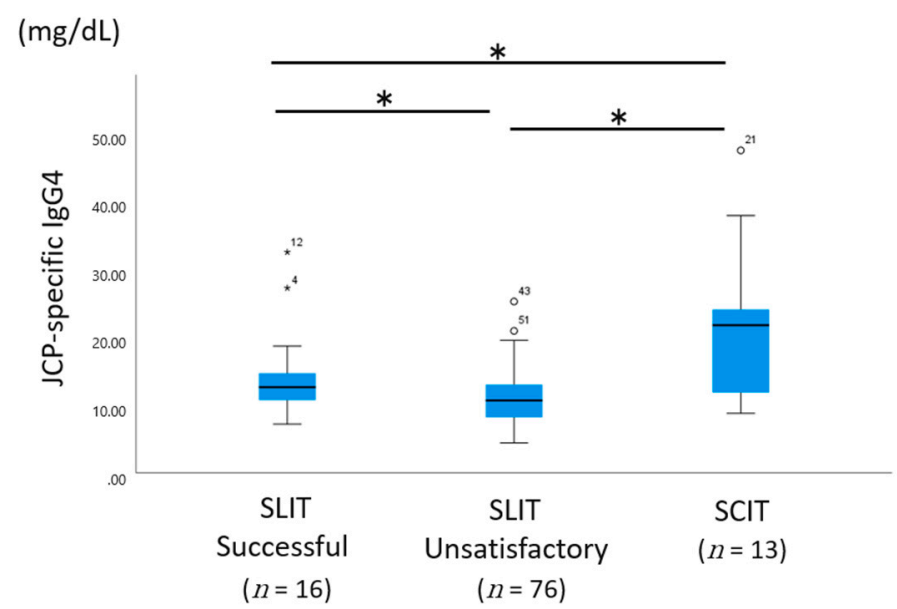

(C)

Figure 1. Cont. 


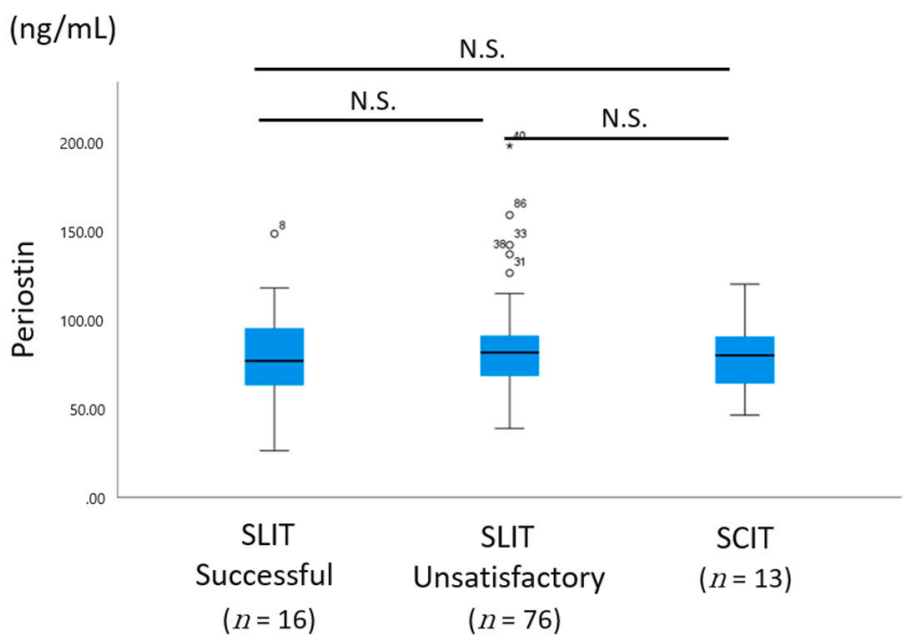

(D)

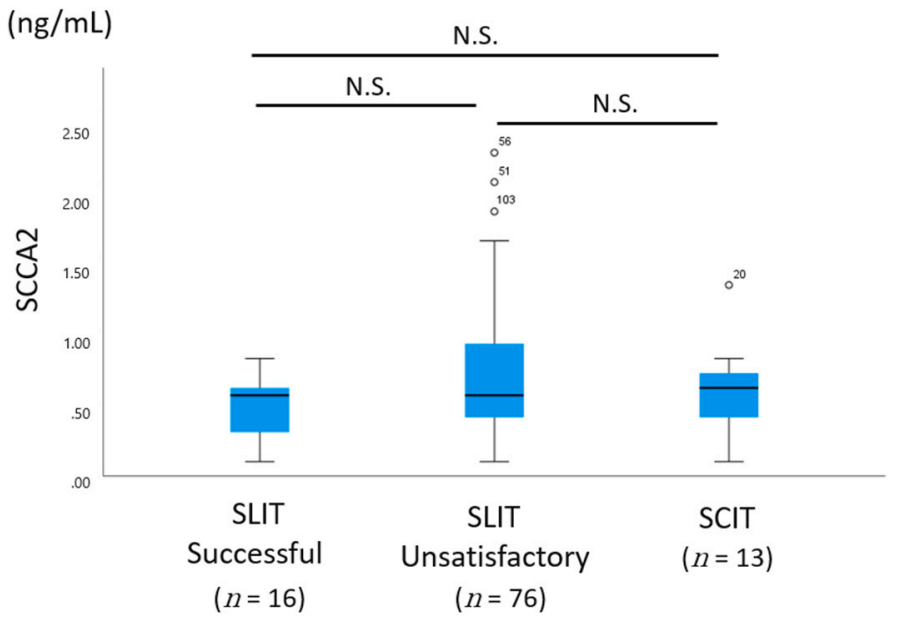

(E)

Figure 1. Serum concentrations of JCP-specific $\operatorname{IgE}(\mathbf{A}), \operatorname{IgG}(\mathbf{B}), \operatorname{IgG} 4(\mathbf{C})$, periostin (D), and SCCA2 (E). ${ }^{*} p<0.05$, N.S.: not significant (Mann-Whitney U test).

\subsection{Serum Levels of Periostin and SCCA2}

There was no significant difference among serum levels of periostin in the SCIT group, the SLIT Successful group, or the SLIT Unsatisfactory group (Figure 1D). There was also no significant difference in SCCA2 among the three groups (Figure 1E).

\subsection{ROC Curves}

Figure 2 shows the sensitivity and specificity obtained by calculating the ROC curves for each serum JCP-specific IgE, IgG, IgG4, periostin, and SCCA2 level and making pairwise comparisons. The area under the curve was 0.653 for serum JCP-specific IgE levels (95\% CI, 0.527-0.779), 0.697 for serum JCP-specific IgG levels (95\% CI, $0.560-0.835), 0.962$ for serum JCP-specific IgG4 levels (95\% CI, 0.923-1.000), 0.486 for serum periostin levels (95\% CI, $0.355-0.617$ ), and 0.417 for serum SCCA2 levels (95\% CI, 0.303-0.531). Our ROC analysis showed the serum JCP-specific IgG4 levels to be the most appropriate for predicting the efficacy of SLIT. Values greater than $989.5 \mathrm{UA} / \mathrm{mL}$ showed the best sensitivity $(93.3 \%)$ and specificity $(94.7 \%)$. 
JCP-specific IgE

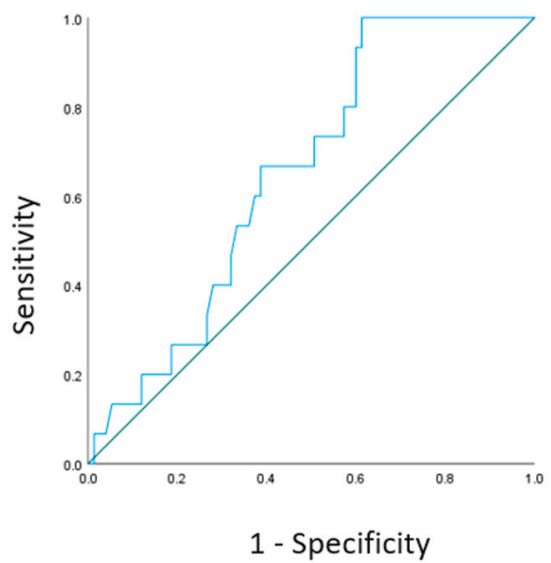

(A)

JCP-specific IgG

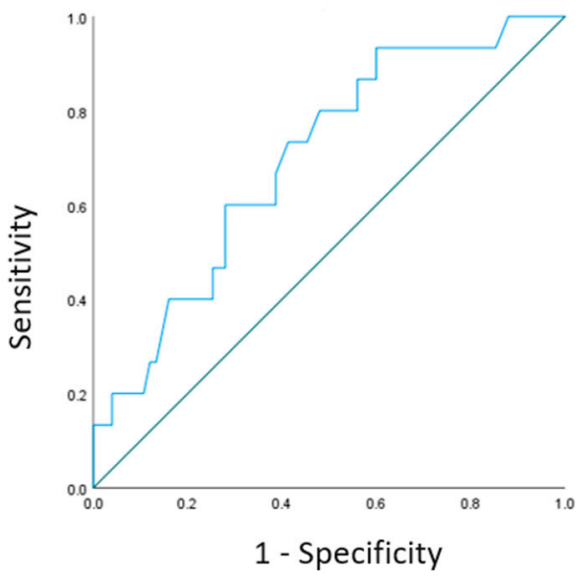

(B)

JCP-specific lgG4

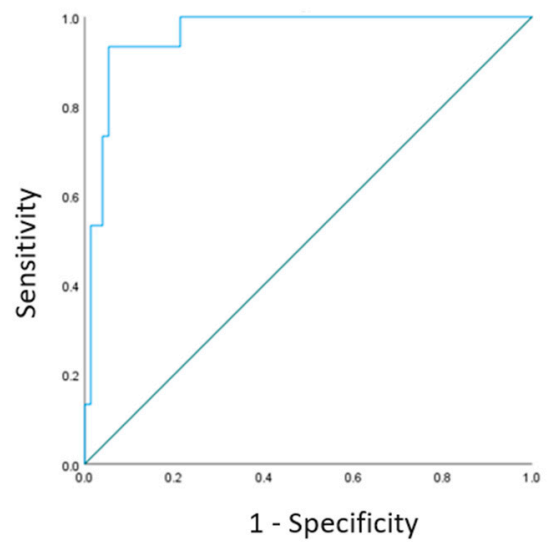

(C)

Figure 2. Cont. 


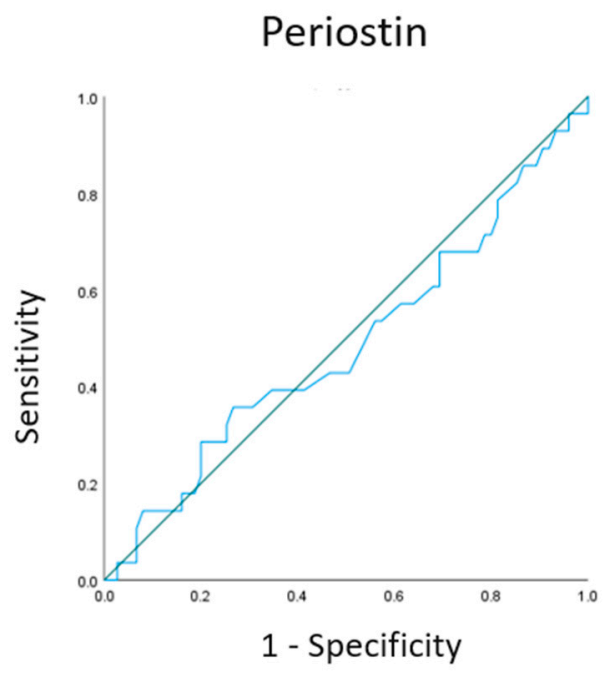

(D)

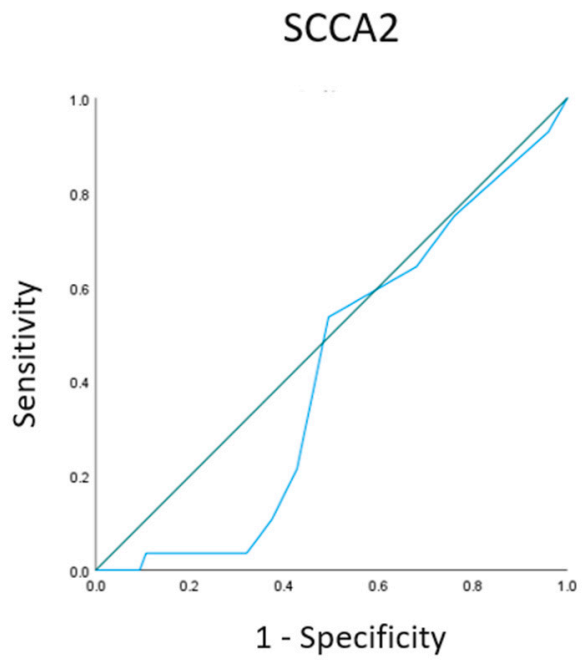

(E)

Figure 2. ROC curves obtained with serum JCP-specific $\operatorname{IgE}(\mathbf{A})$ (decision point, $\leq 100.3 \mathrm{UA} / \mathrm{mL}$; sensitivity, 66.7\%; and specificity), 61.3\%, IgG (B) (decision point, $\leq 11.6 \mathrm{UA} / \mathrm{mL}$; sensitivity, 60.0\%; and specificity, $72.0 \%$ ), IgG4 (C) (decision point, $\leq 989.5 \mathrm{UA} / \mathrm{mL}$; sensitivity, $93.3 \%$; and specificity, 94.7\%), periostin (D) (decision point, $89.5 \leq \mathrm{UA} / \mathrm{mL}$; sensitivity, 28.6\%; and specificity, 80.0\%), SCCA2 (E) (decision point, $\leq 0.550 \mathrm{UA} / \mathrm{mL}$; sensitivity, 53.6\%; and specificity, 50.7\%) by plotting data for them with an effective response to SLIT versus 1-specificity inpatients who showed an ineffective response to SLIT.

\section{Conclusions}

Our study showed serum JCP-specific IgG4 level to be significantly correlated with the clinical efficacy of SLIT. We found that serum JCP-specific IgG4 cutoff levels that exceeded 989.5 UA/mL correlated with an effective clinical response to SLIT, with a sensitivity of $93.3 \%$ and a specificity of $94.7 \%$.

\section{Discussion}

Our study showed the serum JCP-specific IgG4 level to be significantly correlated with the clinical efficacy of SLIT. We found that serum JCP-specific IgG4 cutoff levels greater than $989.5 \mathrm{UA} / \mathrm{mL}$ correlated with an effective clinical response to SLIT, with a sensitivity of $93.3 \%$ and a specificity of $94.7 \%$. Studies for determining biomarkers that predict the efficacy of SLIT against Japanese cedar pollinosis are also very helpful and 
may improve daily QOL, but have not been sufficiently elucidated, especially for JCPspecific immunoglobulins. There have been very few studies that attempt to identify useful biomarkers for evaluating the efficacy of SLIT. Induction of allergen-specific IgG4 has long been regarded as the characteristic immunological feature induced by AIT [8], and several studies have shown that successful use of AIT might induce a substantial increase in allergen-specific IgG4 Abs $[9,10]$. Antigen-specific IgG4 works as a blocking antibody and is involved in the mechanism of SLIT, and serum Cry $\mathrm{j}$ 1-specific IgG4 is elevated after SLIT with JCe pollen extract in association with an increase in IL-10-expressing T cells or $\mathrm{B}$ cells $[11,12]$. Our results were in agreement with these studies: the serum level of JCPspecific IgG4 increased significantly in the SCIT group $(p<0.05)$ and the SLIT Successful group $(p<0.05)$. Moreover, the serum level of JCP-specific IgG4 rose significantly higher in the SCIT group than that of each of the SLIT groups $(p<0.05)$. The immunological effects of SLIT are weaker than those of SCIT [13], so this maybe one of the causes of our result. It is reported that the serum level of JCP-specific IgG4 increased significantly to five times the baseline level after the 2011 JCe pollen season, and this level gradually decreased up to the end of the second season, but still remained higher than at the baseline [3]. It is also reported that JCP-specific IgG4 levels increased significantly after five months of SCIT, with the level gradually increasing if SCIT was continued [14]. There is, therefore, the potential to maintain the serum level of JCP-specific IgG4 at a high level by continuing treatment. AIT has been shown to reduce IgE production [15,16]. It is reported that the JCP-specific IgE level decreased significantly after 17 months of SCIT. However, a 2-year JCe pollen-based SLIT trial showed the JCP-specific IgE titer to be higher after SLIT than the baseline at the end of the first and second JCe pollen season. Similarly, it was found that the serum Cry j1-specific IgE was significantly higher in the SLIT group than in the non-SLIT group after one or two years of SLIT. In our study, the JCP-specific IgE level of the SCIT group was significantly lower than that of the SLIT group $(p<0.05)$. Moreover, it is reported that the JCP-specific IgE level increased significantly by up to twice the baseline level two months after the start of treatment; the elevated level of JCP-specific IgE persisted until the end of the first pollen season; in the second season, this level decreased but, at the end, it was still higher than at baseline [3]. It is therefore possible that the JCPspecific IgE level might decrease if the trial period were extended. Serum IL-17A, SCCA2, periostin, saliva, and pathogenic Th2 cells have been reported as potentially useful clinical biomarkers for evaluating the efficacy of SLIT for JCP [17-19]. Periostin is highly expressed in chronic inflammatory diseases like asthma, chronic rhinosinusitis, atopic dermatitis, and allergic conjunctivitis [20], and has emerged as a useful biomarker of allergic diseases. It is reported that high serum periostin levels $(>30.2 \mathrm{ng} / \mathrm{mL}$ ) are associated with an effective response to house dust mite SLIT, and the degree of improvement as measured by the Rhinoconjunctivitis Quality of Life Questionnaire was correlated with the level of serum periostin [21,22]. In our study, there was no significant difference among serum levels of periostin among the SCIT group, the SLIT Successful group, or the SLIT Unsatisfactory group. SCCA2 is involved in the pathogenesis of several inflammatory diseases: asthma, psoriasis, and atopic dermatitis. It is reported that SCCA2 is useful in aiding diagnosis, estimating clinical severity and disease type, and assessing responses to the treatment of psoriasis and atopic dermatitis. These results suggest that SCCA2 has emerged as a novel biomarker for skin inflammatory diseases [22,23]. The usefulness of SCCA2 as a biomarker for SLIT, however, has hitherto not been evaluated. In our study, there was also no significant difference in SCCA2 among the three groups, as was the case with periostin.

Our study has several limitations. First, it was retrospective and contained only a small number of subjects. Our study is based on cases from our own facility and from other facilities, with a total of fifteen; however, it would require more cases, especially in the SLIT Successful group, to evaluate the results more accurately. Second, the amount of pollen released and climatic conditions vary each year. In 2014, the airborne pollen count was lower than usual, possibly resulting in fewer patients in the pre-symptomatic state group and the SLIT Successful group. Third, the duration of the SLIT experiment was markedly 
shorter than that of the SCIT experiment. The SCIT group was treated for three years, but the SLIT group was treated for only a year because the formulation of SLIT that we used this study had been approved only a year before we embarked on this study. Further studies with serial evaluations of JCP-specific IgE, IgG, and IgG4, including measurements during the JCe pollen release season over several years, are needed to confirm our results. Our results may allow us to speculate that JCP-specific IgG4 plays an important role in the underlying mechanism of SLIT and could act as a predictive marker for clinical remission after SLIT treatment.

Author Contributions: The roles of each of the authors in the preparation of this manuscript are as follows. S.K., A.Y., Y.O. (Yukiko Ogawa), Y.S., T.S., and H.K. worked on data collection. S.I., J.O., R.I., S.K., and T.E. worked on statistical data analysis. K.I., Y.O. (Yoshitaka Okamoto), and N.O. provided insightful comments and suggestions on the manuscript. N.O. is the guarantor of the integrity of the content of the manuscript. All authors have read and agreed to the published version of the manuscript.

Funding: This study was funded by JSPS Kakenhi Grant Number JP17K11363 and the Ministry of Health, Labor, and Welfare of Japan.

Institutional Review Board Statement: The study was conducted according to the guidelines of the Declaration of Helsinki, and approved by the Institutional Review Board of Tohoku Medical and Pharmaceutical University (protocol code 2016-2-2-054 and date of approval 2 February 2016).

Informed Consent Statement: Informed consent was obtained from all subjects involved in the study.

Data Availability Statement: Data is contained within the article.

Conflicts of Interest: The authors declare no conflict of interest.

\section{References}

1. Masuyama, K.; Goto, M.; Takeno, S.; Ohta, N.; Okano, M.; Kamijo, A.; Suzuki, M.; Terada, T.; Sakurai, D.; Horiguchi, S.; et al. Guiding principles of sublingual immunotherapy for allergic rhinitis in Japanese patients. Auris Nasus Larynx 2016, 43, 1-9. [CrossRef]

2. Kikuoka, H.; Kouzaki, H.; Matsumoto, K.; Arai, H.; Yamamoto, S.; Tojima, I.; Shimizu, S.; Miyashita, H.; Ogawa, Y.; Osada, T.; et al. Immunological effects of sublingual immunotherapy with Japanese cedar pollen extract in patients with combined Japanese cedar and Japanese cypress pollinosis. Clin. Immunol. 2019, 210, 108310. [CrossRef] [PubMed]

3. Okamoto, Y.; Okubo, K.; Yonekura, S.; Hashiguchi, K.; Goto, M.; Otsuka, T.; Murata, T.; Nakao, Y.; Kanazawa, C.; Nagakura, H.; et al. Efficacy and Safety of Sublingual Immunotherapy for Two Seasons in Patients with Japanese Cedar Pollinosis. Allergy Immunol. 2015, 166, 177-188. [CrossRef]

4. Fujisawa, T.; Nago, M.; Hiaguchi, Y.; Hosoki, K.; Tokuda, R.; Usui, S.; Masuda, S.; Shinoda, M.; Hashiguchi, A.; Yamaguchi, M. Biomarkers for Allergen Immunotherapy in Cedar Pollinosis. Allergol. Int. 2009, 58, 163-170. [CrossRef]

5. Yonekura, S.; Okamoto, Y.; Sakurai, D.; Okubo, K.; Gotoh, M.; Kaneko, S.; Konno, A. An analysis of factors related to the effect of sublingual immunotherapy on Japanese cedar pollen induced allergic rhinitis. Allergol Int. 2018, 67, 201-208. [CrossRef]

6. Ishida, A.; Ohta, N.; Suzuki, Y.; Kakehata, S.; Okubo, K.; Ikeda, H.; Shiraishi, H.; Izuhara, K. Expression of pendrin and periostin in allergic rhinitis and chronic rhinosinusitis. Allergol. Int. 2012, 61, 589-595. [CrossRef] [PubMed]

7. Nagao, M.; Inagaki, S.; Kawano, T.; Azuma, Y.; Nomura, N.; Noguchi, Y.; Ohta, S.; Kawaguchi, A.; Odajima, H.; Ohya, Y.; et al. SCCA2 is a reliable biomarker for evaluating pediatric atopic dermatitis. J. Allergy Clin. Immunol. 2018, 141, 1934-1936. [CrossRef]

8. Şahin, E.; Bafaqeeh, S.A.; Güven, S.G.; Çetinkaya, E.A.; Muluk, N.B.; Coşkun, Z.O.; Lopatin, A.; Kar, M.; Pinarbasli, M.O.; Cingi, C. Mechanism of action of allergen immunotherapy. Am. J. Rhinol. Allergy 2016, 30, 1-3. [CrossRef]

9. Wachholz, P.A.; Soni, N.K.; Till, S.J.; Durham, S.R. Inhibition of allergen-IgE binding to B cells by IgG antibodies after grass pollen immunotherapy. J. Allergy Clin. Immunol. 2003, 112, 915-922. [CrossRef]

10. Shamji, M.H.; Ljørring, C.; Francis, J.N.; Calderon, M.; Larche, M.; Kimber, I.; Frew, A.J.; Ipsen, H.; Lund, K.; Würtzen, P.A.; et al. Functional rather than immunoreactive levels of IgG4 correlate closely with clinical response to grass pollen immunotherapy. Allergy 2012, 67, 217-226. [CrossRef]

11. Horiguchi, S.; Okamoto, Y.; Yonekura, S.; Okawa, T.; Yamamoto, H.; Kunii, N.; Sakurai, D.; Fujimura, T.; Nakazawa, K.; Yasueda, H. A randomized controlled trial of sublingual immunotherapy for Japanese cedar pollinosis. Int. Arch. Allergy Immunol. 2008, 146, 76-84. [CrossRef]

12. Sakurai, S.; Yonekura, S.; Iinuma, T.; Morimoto, Y.; Mita, Y.; Arai, T.; Suzuki, S.; Okuma, Y.; Kaneko, S.; Okamoto, Y. Sublingual immunotherapy for allergic rhinitis: Subjective versus objective tools to evaluate its success. Rhinology 2016, 54, 221-230. [CrossRef] [PubMed] 
13. Burks, A.W.; Calderon, M.A.; Casale, T.; Cox, L.; Demoly, P.; Jutel, M.; Nelson, H.; Akdis, C.A. Update on allergy immunotherapy: American Academy of Allergy, Asthma \& Immunology/European Academy of Allergy and Clinical Immunology/PRACTALL consensus report. J. Allergy Clin Immunol. 2013, 131, 1288-1296. [PubMed]

14. Inuo, C.; Ando, H.; Tanaka, K.; Nakajima, Y.; Tsuge, I.; Urisu, A.; Kondo, Y. Long-term immunological effects of Japanese cedar pollen-based subcutaneous immunotherapy. Allergol. Int. 2018, 67, 408-410. [CrossRef]

15. Varga, E.M.; Francis, J.N.; Zach, M.S.; Klunker, S.; Aberer, W.; Durham, S.R. Time course of serum inhibitory activity for facilitated allergen-IgE binding during bee venom immunotherapy in children. Clin. Exp. Allergy 2009, 39, 1353-1357. [CrossRef] [PubMed]

16. Gleich, G.J.; Zimmermann, E.M.; Henderson, L.L.; Yunginger, J.W. Effect of immunotherapy on immunoglobulin E and immunoglobulin G antibodies to ragweed antigens: A six-year prospective study. J. Allergy Clin. Immunol. 1982, 70, $261-271$. [CrossRef]

17. Haruna, T.; Kariya, S.; Fujiwara, T.; Yuta, A.; Higaki, T.; Zhao, P.; Ogawa, Y.; Kanai, K.; Hirata, Y.; Oka, A.; et al. Role of whole saliva in the efficacy of sublingual immunotherapy in seasonal allergic rhinitis. Allergol. Int. 2019, 68, 82-89. [CrossRef] [PubMed]

18. Sakashita, M.; Yamada, T.; Imoto, Y.; Hirota, T.; Tamari, M.; Ito, Y.; Kubo, S.; Osawa, Y.; Takahashi, N.; Fujieda, S. Long-term sublingual immunotherapy for Japanese cedar pollinosis and the levels of IL-17A and complement components 3a and 5a. Cytokine 2015, 75, 181-185. [CrossRef] [PubMed]

19. Iinuma, T.; Okamoto, Y.; Morimoto, Y.; Arai, T.; Sakurai, T.; Yonekura, S.; Sakurai, D.; Hirahara, K.; Nakayama, T. Pathogenicity of memory Th2 cells is linked to stage of allergic rhinitis. Allergy 2018, 73, 479-489. [CrossRef]

20. Izuhara, K.; Nunomura, S.; Nanri, Y.; Ogawa, M.; Ono, J.; Mitamura, Y.; Yoshihara, T. Periostin in inflammation and allergy. Cell Mol. Life Sci. 2017, 74, 4293-4303. [CrossRef]

21. Hoshino, M.; Akitsu, K.; Kubota, K.; Ohtawa, J. Serum Periostin as a Biomarker for Predicting Clinical Response to House Dust Mite Sublingual Immunotherapy in Allergic Rhinitis. J. Allergy Clin. Immunol. Pract. 2020, 9, 1864-1870. [CrossRef] [PubMed]

22. Izuhara, K.; Yamaguchi, Y.; Ohta, S.; Nunomura, S.; Nanri, Y.; Azuma, Y.; Nomura, N.; Noguchi, Y.; Aihara, M. Squamous Cell Carcinoma Antigen 2 (SCCA2, SERPINB4): An Emerging Biomarker for Skin Inflammatory Diseases. Int. J. Mol. Sci. 2018, 19, 1102. [CrossRef] [PubMed]

23. Nomura, Y.; Okubo, K.; Nakamura, T.; Sawaki, S.; Kitagou, H.; Idei, N.; Kaneko, S.; Kobayashi, S.; Tanaka, Y.; Okamoto, Y. Long-term treatment of Japanese cedar pollinosis with Japanese cedar pollen SLIT drops and persistence of treatment effect: A post-marketing clinical trial. Allergol. Int. 2021, 70, 96-104. [CrossRef] [PubMed] 